\title{
CLOSE ASSOCIATION OF AN EXTREME-ULTRAVIOLET SUNSPOT PLUME WITH DEPRESSIONS IN THE SUNSPOT RADIO EMISSION
}

\author{
JEFFREY W. BROSIUS \\ Catholic University of America at NASA Goddard Space Flight Center, Code 682, Greenbelt, MD 20771 \\ AND \\ STEPhen M. White \\ Department of Astronomy, University of Maryland, College Park, MD 20742 \\ Received 2003 July 31; accepted 2003 October 1
}

\begin{abstract}
We obtained coordinated observations of the large sunspot in NOAA Region 8539 on 1999 May 9 and 13 with the Very Large Array and three instruments (CDS, EIT, MDI) aboard the Solar and Heliospheric Observatory satellite. The EUV observations reveal a plume in the sunspot umbra on both observing dates. The plume appears brightest in emission lines formed at temperatures between $1.6 \times 10^{5}$ and $5.0 \times 10^{5} \mathrm{~K}$. Radio emission from the sunspot umbra is dominated by thermal gyroemission from the plume, which accounts for radio brightness temperatures $<1 \times 10^{6} \mathrm{~K}$ in the umbra on both dates, as well as umbral brightness temperature depressions in the 4.535 and $8.065 \mathrm{GHz}$ observations on May 13. A compact $14.665 \mathrm{GHz}$ source persists near the umbra/penumbra boundary during our observing period, indicating a long-lived, compact flux tube with coronal magnetic field strength of at least $1748 \mathrm{G}$. It occurs in a portion of the sunspot that appears very dark in EUV emission.
\end{abstract}

Subject headings: Sun: corona — Sun: magnetic fields — Sun: radio radiation — Sun: transition region — Sun: UV radiation - sunspots

On-line material: color figures

\section{INTRODUCTION}

It is well known that the centimetric radio emission observed from sunspots is dominated by thermal gyroresonance emission (e.g., Kundu 1965; Zheleznyakov 1970; White \& Kundu 1997, and references therein; White 2004). Thermal gyroresonance emission (or, more simply, gyroemission) occurs where the radio observing frequency is a low harmonic (typically second or third) of the electron gyrofrequency in the radio source. For example, second and third harmonic gyroemission at a radio observing frequency of $5 \mathrm{GHz}(6 \mathrm{~cm}$ wavelength) originates in plasma with magnetic field strengths of 900 and $600 \mathrm{G}$, respectively. Similarly, at a frequency of $15 \mathrm{GHz}(2 \mathrm{~cm})$ the field strengths corresponding to second and third harmonic gyroemission are 2700 and $1800 \mathrm{G}$. The measurement of brightness temperatures $\sim 1-3 \mathrm{MK}$ in and around sunspots indicates that magnetic field strengths appropriate for gyroemission are present in the corona and, hence, provides valuable information about an important but elusive quantity in solar physics: the coronal magnetic field.

Sunspots appear very different at extreme ultraviolet (EUV) than they do at radio wavelengths. This is because the EUV radiation depends predominantly on the temperature and emission measure of the emitting plasma, while radio gyroemission depends predominantly on the magnetic field in its source region. The EUV radiation is due to optically thin line emission from collisionally excited ions of trace elements, and appears brightest where the emission measure (and hence the optical depth) is greatest. Since sunspots contain relatively small amounts of plasma at coronal temperatures (e.g., Nindos et al. 2000), they appear dark in coronal emission lines.

Model calculations of the radio emission from a sunspot with a simple dipole magnetic field indicate that the radio source should exhibit a relative intensity depression on the side of the spot closest to solar disk center and a relative intensity peak on the side of the spot closest to the solar limb (Gelfreikh \& Lubyshev 1979; Brosius \& Holman 1989). This occurs because the gyroresonance opacity decreases with decreasing angle between the magnetic field and the line of sight. The corona is optically thin where the angle is small, so radio emission is seen from deeper in the solar atmosphere, where the plasma is cooler. Further, projection effects produce a limbward shift in the apparent location of a radio source located at some height above the photospheric sunspot.

Alissandrakis \& Kundu (1982) used the Westerbork Synthesis Radio Telescope (WSRT) to observe a sunspot at $5 \mathrm{GHz}$ on six successive days. Their intensity maps revealed a ring structure around the sunspot umbra, with an intensity depression toward the center of the ring. Such a depression could be explained by angular effects when the spot was near disk center; however, because the location of the intensity depression as well as the overall structure of the radio emission did not change much during the six days of observations, Alissandrakis \& Kundu concluded that the radio emission from the intensity depression must originate in relatively cool plasma. Further support for the presence of cool material in this sunspot's umbra was subsequently reported by Strong, Alissandrakis, \& Kundu (1984) based on modeling of the coordinated radio and soft X-ray observations obtained with the X-Ray Polychromator (XRP) aboard the Solar Maximum Mission (SMM) satellite. Both Alissandrakis \& Kundu (1982) and Strong et al. (1984) mention a possible association of the cool umbral plasma seen in the $5 \mathrm{GHz}$ radio emission with sunspot plumes (Foukal et al. 1974).

Foukal et al. (1974) were the first to note the relative brightness of sunspots in EUV line emission formed at transition region temperatures. This was based on solar active region 
and sunspot observations obtained with the SO55 spectroheliometer on board Skylab. Sources of enhanced sunspot transition region emission were termed "plumes," and a subsequent study of the 22 largest spots observed with the SO55 revealed that they all exhibited plumes (Foukal 1976). Noyes et al. (1985) presented an averaged plume emission-line spectrum and showed that the relative intensity enhancement in plumes is greatest for lines formed at temperatures between about $1.6 \times 10^{5}$ and $4.0 \times 10^{5} \mathrm{~K}(5.2 \leq \log T \leq 5.6)$. More recent studies of sunspots with the Coronal Diagnostic Spectrometer (CDS) aboard the Solar and Heliospheric Observatory $(\mathrm{SOHO})$ satellite provide further evidence that most, if not all, sunspots exhibit plumes (Fludra et al. 1997; Maltby et al. 1998; Brynildsen et al. 1999).

Numerous authors have noted that the observed radio brightness temperature distribution above sunspots does not conform to that expected from either simple dipole model calculations (Gary \& Hurford 1987; Brosius et al. 1992; White, Kundu, \& Gopalswamy 1992; Vourlidas, Bastian, \& Aschwanden 1997) or photospheric magnetic field extrapolations (Alissandrakis, Kundu, \& Lantos 1980; Gopalswamy et al. 1996; Nindos et al. 1996; Lee et al. 1998; Brosius et al. 2002). For example, Gary \& Hurford (1987) found that peak sunspot radio brightness temperatures were displaced toward the center of an active region in all 16 observing frequencies at which one-dimensional scans were obtained with the Owens Valley frequency-agile interferometer during a partial solar eclipse. They mention the possibility of cool plume plasma dominating the radio emission on the side of the sunspots away from the center of the active region. Brosius et al. (1992) and White et al. (1992) report similar displacements of radio brightness temperature peaks toward the center of an active region in Very Large Array (VLA) maps and mention possible contributions from temperature and/or density inhomogeneities. Alternatively, Alissandrakis et al. (1980), Gopalswamy et al. (1996), Nindos et al. (1996), Lee et al. (1998), and Brosius et al. (2002) conclude that coronal electric currents play an important role in the magnetic fields of sunspots. Within the context of the present work, however, it should be mentioned that none of the above references specifically include plumes in their sunspot models.

Coordinated EUV and radio observations have provided powerful diagnostics of the plasma and magnetic field in solar active regions (e.g., Brosius 2004; Brosius et al. 1993, 1997, 2002; White 1999; White et al. 2000; Zhang et al. 2001). It is, therefore, expected that coordinated EUV and radio observations of sunspots will provide similarly powerful diagnostics. Although sunspots have been independently well observed at EUV and radio wavelengths, they have not been well observed simultaneously in both. Thus, to date, the effect of sunspot plumes on the observed radio emission could at best be speculated. Only with coordinated EUV and radio imagery can the location of sunspot plumes within radio emission maps be unambiguously determined. Here we report results of one such recent set of coordinated observations. We obtained $\mathrm{SOHO}$ CDS EUV and coordinated VLA radio observations of NOAA region 8539 on 1999 May 9 and 13 as part of SOHO Joint Observing Program (JOP) 100. In $\S 2$ we describe our observations and data reduction procedures, in $\S 3$ we present our results, in $\S 4$ we discuss the implications of our work, and in $\S 5$ we summarize our conclusions.

\section{OBSERVATIONS AND DATA REDUCTION}

We observed NOAA Active Region 8539 with the CDS and the VLA from 1400 to 2400 UT on 1999 May 9 and from 2000 to 2400 UT on 1999 May 13. The region contained a large sunspot, located at N22 ${ }^{\circ} 30^{\circ}\left(-450^{\prime \prime},+400^{\prime \prime}\right.$ in solar disk coordinates) around 1900 UT on May 9 and at $\mathrm{N} 20^{\circ}$ W $21^{\circ}\left(+320^{\prime \prime},+380^{\prime \prime}\right)$ around 1900 UT on May 13.

CDS (Harrison et al. 1995) on board the SOHO satellite contains a Normal Incidence Spectrometer (NIS) that can be used to obtain instantaneous stigmatic EUV spectra within its $308-381 \AA$ (NIS-1) and 513-633 $\AA$ (NIS-2) wavebands for each of 143 1".68 spatial pixels along its $4^{\prime}$ long slit. We constructed $4^{\prime} \times 4^{\prime}$ raster images of our target region by obtaining NIS-1 and NIS-2 spectra from 60 successive slit pointings with the $4^{\prime \prime}$ wide slit. Spectra were compressed (averaged) along the slit by a factor of 4 in order to limit the total raster time to two hr. In this mode the CDS spatial resolution was nominally 4."0 0 6".7. We obtained five $2 \mathrm{hr}$ rasters of AR 8539 on May 9 and two 2 hr rasters on May 13.

The CDS spectra were processed and calibrated using standard SolarSoftware IDL procedures. In particular, in order to get intensity arrays for the lines used in this work (see Figs. 1 and 2 and Tables 1 and 2), we fitted profiles of 33 lines in 12 NIS-2 channels and of six lines in 2 NIS-1 channels. The lines were fitted with "broadened Gaussians"1 in order to accommodate the profile broadening and asymmetry that resulted from the temporary loss of SOHO attitude control in 1998. Since broadening is worse in NIS-1 than NIS-2, we use mostly NIS-2 lines in this work. The integrated intensity of an NIS-2 line can be written $\mathcal{I}=1.485 \mathrm{Aw}$, where $A$ is the amplitude and $w$ is the "broadened width" (effectively the FWHM).

We also obtained full-disk, 2."62 pixel images of He II at 304 $\AA, \mathrm{Fe}$ IX/X at $171 \AA$, Fe XII at $195 \AA$, and Fe XV at $284 \AA$ with the $\mathrm{SOHO}$ Extreme-ultraviolet Imaging Telescope (EIT) around 1900 UT on both dates. These were used to fine-tune the CDS pointing (which by itself is accurate to only about $15^{\prime \prime}$ ) by visually co-aligning CDS intensity images with EIT images in lines formed at comparable temperatures. It is worth mentioning that some distortion was evident in the CDS images relative to their EIT snapshot counterparts owing to the $2 \mathrm{hr}$ period during which the raster images were constructed. We found that we could compensate for this by adjusting the CDS pixel size from its nominal value of $4^{\prime \prime} 0 \times 66^{\prime \prime} 7$ to $4{ }^{\prime \prime} 3 \times 6$.".7, an increase of $6.7 \%$ in the $x$-direction. We also used the EIT images to look for loops or other structures that might be associated with features in the radio imagery.

We co-aligned and averaged the five CDS rasters from May 9 to obtain average EUV images (Fig. 1), and did the same with the two CDS rasters from May 13 (Fig. 2). In the analysis that follows we use these average images to focus on the $2^{\prime} \times 2^{\prime}$ area around the sunspot on each observing date.

We obtained full disk, 1".98 pixel continuum intensity images and longitudinal magnetograms with $\mathrm{SOHO}^{\mathrm{s}} \mathrm{s}$ Michelson Doppler Imager (MDI) around 1900 UT on both observing dates. The intensity images yield the location of the sunspot umbra and penumbra, while the magnetograms yield the strength and orientation of the photospheric magnetic field.

\footnotetext{
${ }^{1}$ CDS Software Note 53, available at http://solg2.bnsc.rl.ac.uk/software/ notes.shtml.
} 


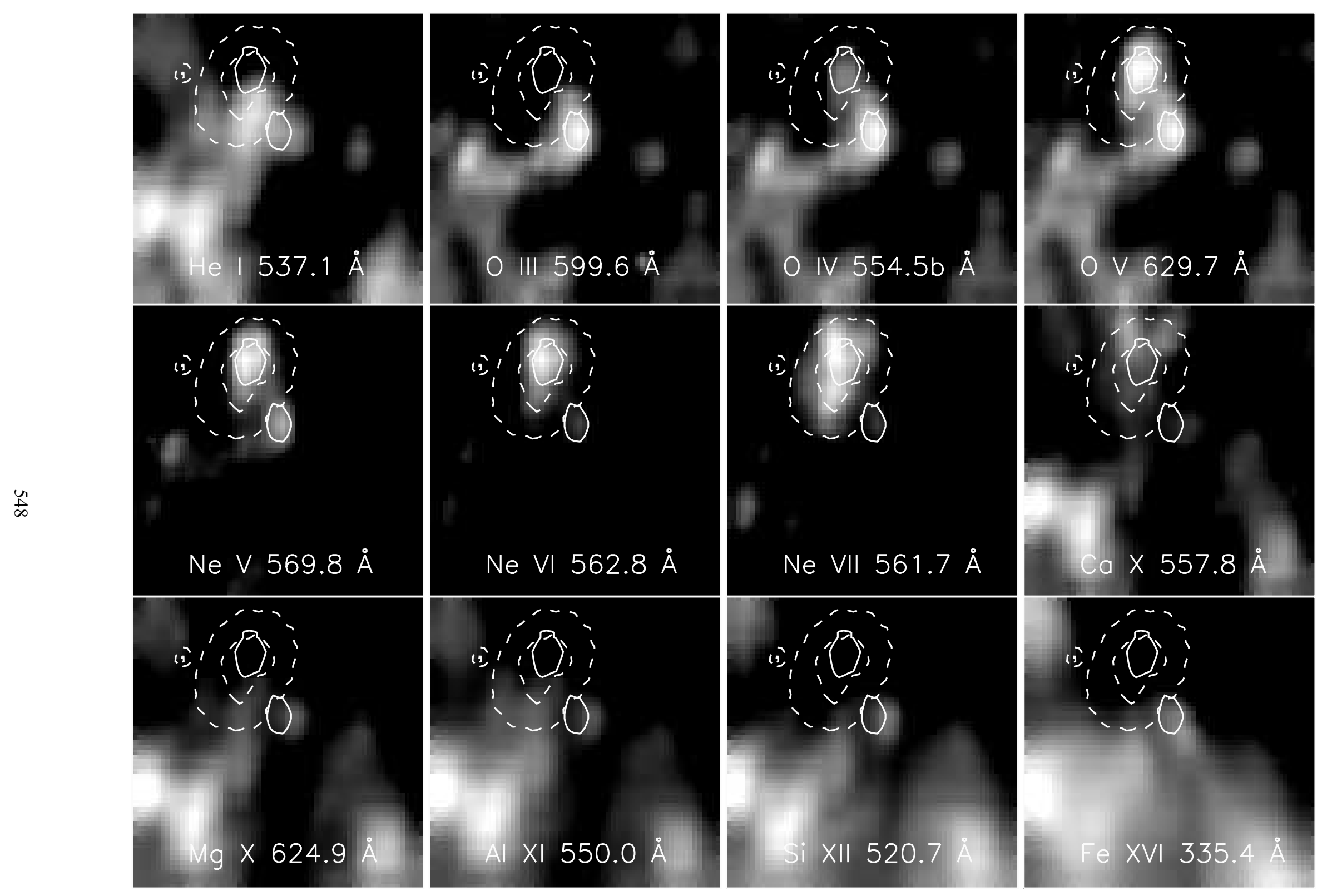

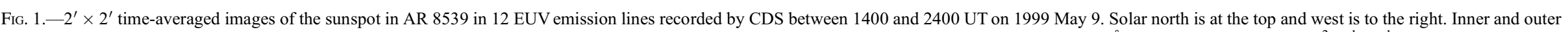

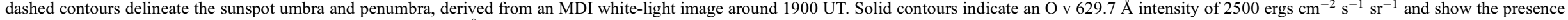
of the plume within the sunspot umbra. The $\mathrm{O}$ iv line at $554.5 \AA$ is a blend of four closely spaced $\mathrm{O}$ iv lines. [See the electronic edition of the Journal for a color version of this figure.] 

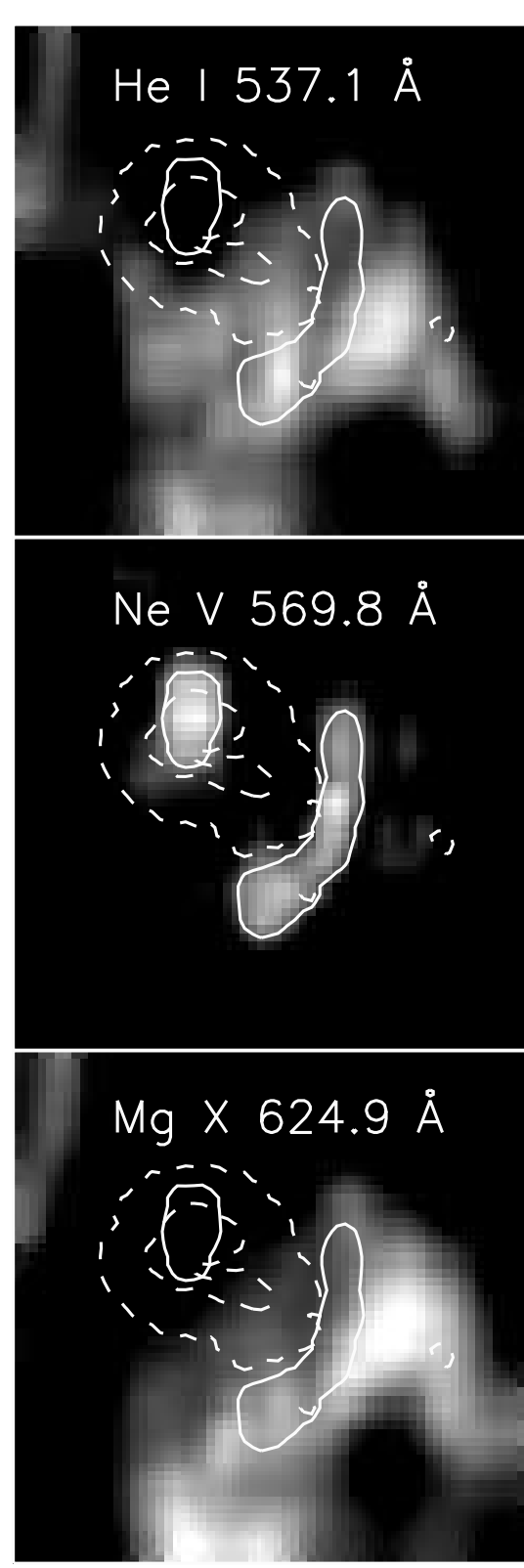
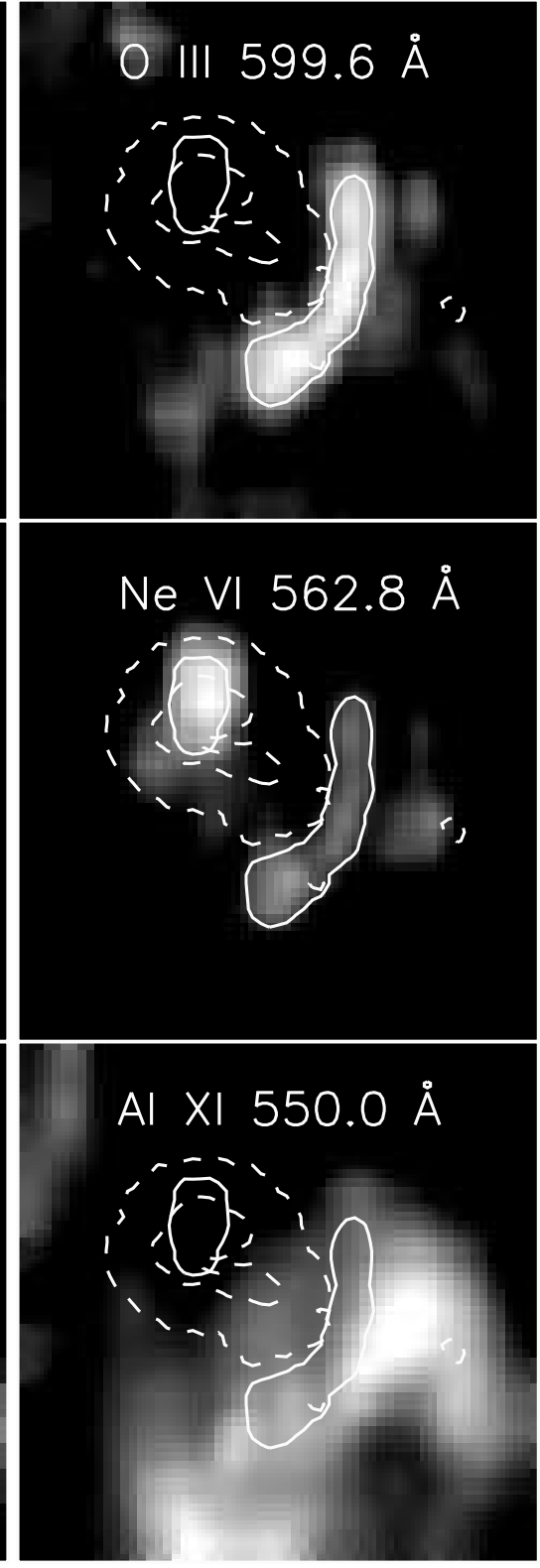

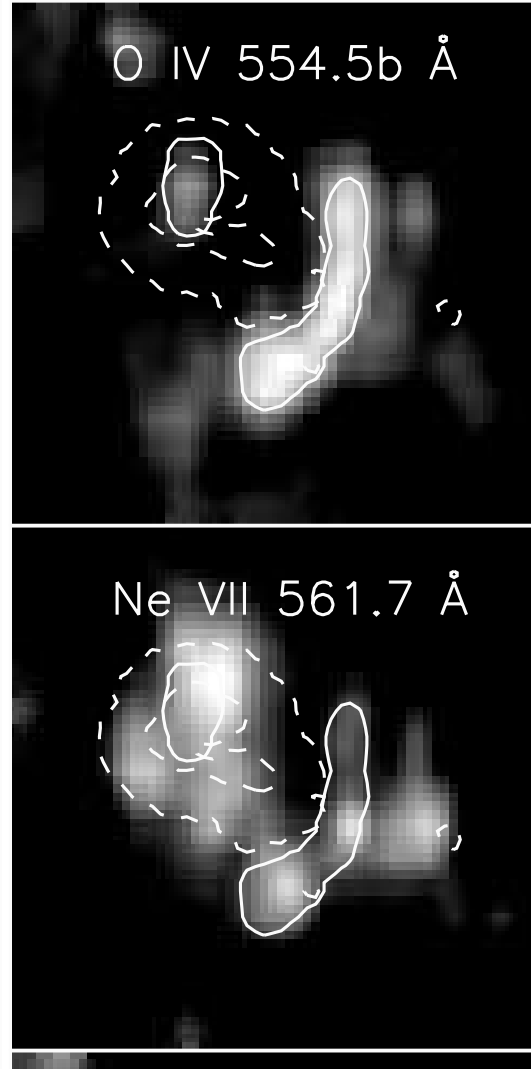

Si XII $520.7 \AA$

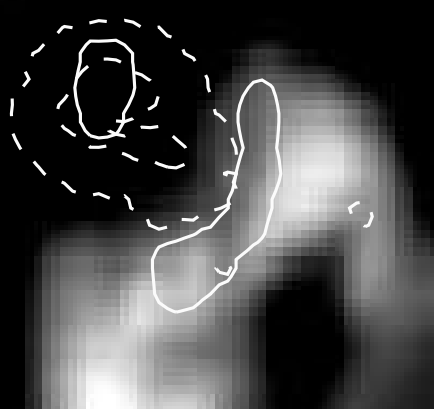

O V $629.7 \AA$

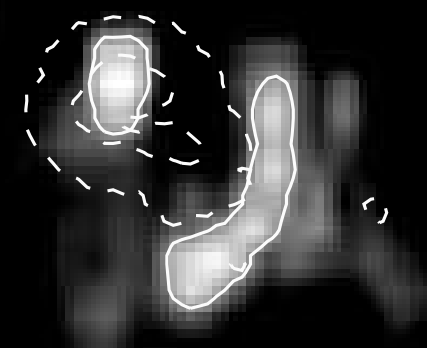

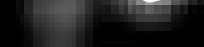

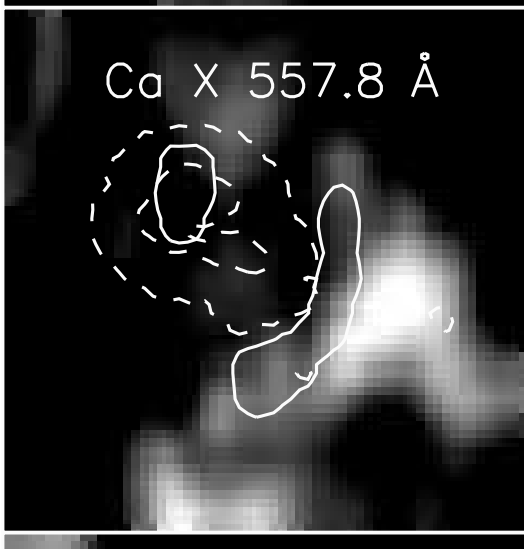

Fe XVI $335.4 \AA$ ,

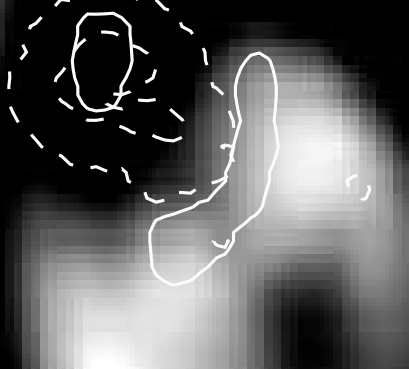

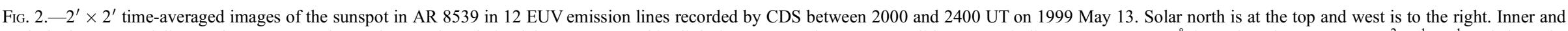

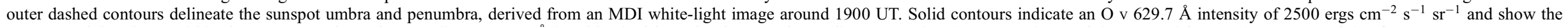
presence of the plume within the sunspot umbra. The $\mathrm{O}$ Iv line at $554.5 \AA$ is a blend of four closely spaced $\mathrm{O}$ Iv lines. [See the electronic edition of the Journal for a color version of this figure.] 
TABLE 1

EUV Line Intensities in the Plume, Sunspot, and Surroundings

\begin{tabular}{|c|c|c|c|c|c|}
\hline \multirow[b]{2}{*}{ LiNE } & \multirow[b]{2}{*}{$\log T_{\max }$} & \multicolumn{3}{|c|}{1999 May 9 Average Intensities } & \multirow[b]{2}{*}{ MaX } \\
\hline & & Plume & Sunspot & Region & \\
\hline He I $522.2 \ldots \ldots \ldots \ldots . . . .$. & 4.0 & $98.3 \pm 19.5$ & $119.6 \pm 8.2$ & $107.1 \pm 1.7$ & $354.0 \mathrm{r}$ \\
\hline He I $537.1 \ldots \ldots \ldots \ldots \ldots$. & & $324.5 \pm 66.6$ & $376.6 \pm 22.0$ & $322.3 \pm 4.6$ & $1066.8 \mathrm{r}$ \\
\hline He I $584.3 \ldots \ldots \ldots \ldots \ldots . . .$. & & $4784.5 \pm 765.1$ & $5314.2 \pm 240.7$ & $4387.3 \pm 51.2$ & $10280.5 \mathrm{r}$ \\
\hline $\mathrm{O}$ III $525.8 \ldots \ldots \ldots \ldots \ldots . . .$. & 4.9 & $32.8 \pm 4.9$ & $34.2 \pm 4.4$ & $44.2 \pm 1.9$ & $175.1 \mathrm{r}$ \\
\hline 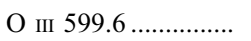 & & $53.1 \pm 7.6$ & $56.1 \pm 7.1$ & $70.2 \pm 2.3$ & $342.3 \mathrm{r}$ \\
\hline 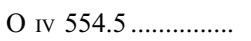 & 5.2 & $921.5 \pm 55.5$ & $534.8 \pm 58.2$ & $474.9 \pm 15.1$ & $2109.4 \mathrm{r}$ \\
\hline 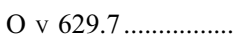 & 5.4 & $3323.8 \pm 434.2$ & $1523.0 \pm 79.3$ & $967.0 \pm 16.3$ & $3801.3 \mathrm{p}$ \\
\hline Ne IV $542.0 \ldots \ldots \ldots \ldots . . . .$. & 5.2 & $28.7 \pm 1.7$ & $15.1 \pm 1.4$ & $9.2 \pm 0.2$ & $56.4 \mathrm{r}$ \\
\hline $\mathrm{Ne} v 569.8 \ldots \ldots \ldots \ldots \ldots . . . .$. & 5.5 & $100.7 \pm 14.2$ & $42.5 \pm 4.7$ & $16.0 \pm 0.7$ & $134.9 \mathrm{p}$ \\
\hline $\mathrm{Ne}$ v $572.2 \ldots \ldots \ldots \ldots \ldots$ & & $175.9 \pm 23.4$ & $72.2 \pm 5.2$ & $29.7 \pm 0.9$ & $231.7 \mathrm{p}$ \\
\hline Ne vi $558.6 \ldots \ldots \ldots \ldots . . .$. & 5.6 & $225.1 \pm 31.2$ & $99.1 \pm 5.4$ & $33.9 \pm 1.1$ & $320.7 \mathrm{p}$ \\
\hline Ne vi $562.8 \ldots \ldots \ldots \ldots . . .$. & & $412.9 \pm 63.7$ & $175.5 \pm 10.8$ & $58.6 \pm 2.3$ & $594.8 \mathrm{p}$ \\
\hline Ne VII $561.7 \ldots \ldots \ldots \ldots . .$. & 5.7 & $78.6 \pm 9.7$ & $46.5 \pm 1.0$ & $13.6 \pm 0.5$ & $111.5 \mathrm{p}$ \\
\hline $\mathrm{Ca} \times 557.8 \ldots \ldots \ldots \ldots \ldots$ & 5.9 & $87.0 \pm 10.8$ & $74.8 \pm 5.5$ & $64.2 \pm 3.1$ & $238.4 \mathrm{r}$ \\
\hline 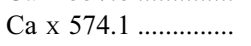 & & $42.1 \pm 5.6$ & $40.8 \pm 0.7$ & $36.8 \pm 1.8$ & $132.2 \mathrm{~s}$ \\
\hline Mg Ix $368.1 \ldots \ldots \ldots \ldots . .$. & 6.0 & $236.0 \pm 23.1$ & $274.1 \pm 18.0$ & $288.3 \pm 18.1$ & $1009.5 \mathrm{r}$ \\
\hline $\operatorname{Mg} \times \quad 624.9 \ldots \ldots \ldots \ldots$ & 6.1 & $154.3 \pm 31.2$ & $225.3 \pm 16.8$ & $329.1 \pm 13.2$ & $1202.7 \mathrm{r}$ \\
\hline $\mathrm{Al}$ XI $550.0 \ldots \ldots \ldots \ldots$ & 6.2 & $51.2 \pm 6.6$ & $67.1 \pm 5.0$ & $92.5 \pm 3.1$ & $351.6 \mathrm{r}$ \\
\hline 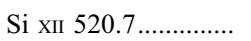 & 6.3 & $60.1 \pm 16.8$ & $147.3 \pm 22.0$ & $439.5 \pm 22.2$ & $1615.3 \mathrm{r}$ \\
\hline Fe xvi $335.4 \ldots \ldots \ldots \ldots . . . .$. & 6.4 & $312.8 \pm 70.8$ & $652.7 \pm 94.8$ & $1851.0 \pm 126.4$ & $5892.7 \mathrm{r}$ \\
\hline
\end{tabular}

Notes.-Line wavelengths are in $\AA$. $T_{\max }$ is the temperature at which a given line's fractional ion abundance is maximized, i.e., the line's "formation temperature"; values here are adopted from Mazzotta et al. (1998) as incorporated into the CHIANTI database (Dere et al. 1997; Young, Landi, \& Thomas 1998; Landi et al. 1999). Line intensities are in ergs $\mathrm{cm}^{-2} \mathrm{~s}^{-1} \mathrm{sr}^{-1}$. The O Iv line is a blend of four closely spaced $\mathrm{O}$ Iv lines. Average sunspot intensities include the plume, and average region intensities include the sunspot. Uncertainties on the tabulated averages encompass the maximum and minimum averages from the five rasters and, hence, the maximum range due to time variability. The maximum intensity ("Max") corresponds to the maximum value measured in any CDS spatial pixel within the $2^{\prime} \times 2^{\prime}$ FOV; "p" means "in the plume," "s" means "in the sunspot," and "r" means "in the region."

TABLE 2

EUV Line Intensities in the Plume, Sunspot, and Surroundings

\begin{tabular}{|c|c|c|c|c|}
\hline \multirow[b]{2}{*}{ LiNE } & \multicolumn{3}{|c|}{1999 May 13 Average Intensities } & \multirow[b]{2}{*}{ Max } \\
\hline & Plume & Sunspot & Region & \\
\hline 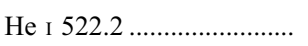 & $51.4 \pm 2.0$ & $121.9 \pm 1.1$ & $118.1 \pm 1.4$ & $519.1 \mathrm{r}$ \\
\hline He г 537.1 & $154.6 \pm 12.7$ & $410.2 \pm 7.8$ & $374.4 \pm 2.3$ & $1527.6 \mathrm{r}$ \\
\hline 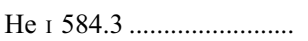 & $2745.4 \pm 209.2$ & $6512.6 \pm 86.0$ & $5541.1 \pm 0.4$ & $11680.0 \mathrm{r}$ \\
\hline 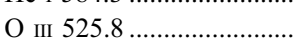 & $27.4 \pm 1.7$ & 34.2 & 48.0 & $267.0 \mathrm{r}$ \\
\hline 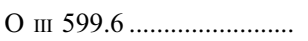 & $50.4 \pm 2.2$ & $64.7 \pm 1.6$ & $87.8 \pm 1.0$ & $494.5 \mathrm{r}$ \\
\hline 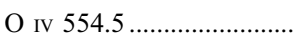 & $1086.6 \pm 46.5$ & $589.6 \pm 1.8$ & $576.2 \pm 1.6$ & $2978.6 \mathrm{r}$ \\
\hline 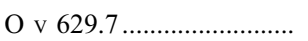 & $3775.1 \pm 3.8$ & $1579.6 \pm 11.3$ & $1069.2 \pm 10.6$ & $5201.1 \mathrm{p}$ \\
\hline 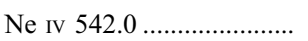 & $31.4 \pm 1.2$ & $15.5 \pm 0.5$ & $11.5 \pm 0.1$ & $75.3 \mathrm{r}$ \\
\hline 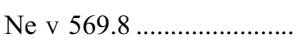 & $122.9 \pm 0.7$ & $45.0 \pm 0.1$ & $21.7 \pm 0.7$ & $182.7 \mathrm{r}$ \\
\hline 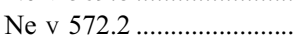 & $212.7 \pm 1.1$ & $90.3 \pm 8.2$ & $41.2 \pm 0.1$ & $276.7 \mathrm{p}$ \\
\hline 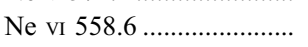 & $221.1 \pm 10.6$ & $101.4 \pm 0.3$ & $42.8 \pm 0.6$ & $303.8 \mathrm{p}$ \\
\hline 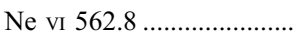 & $442.5 \pm 15.2$ & $189.5 \pm 1.3$ & $77.0 \pm 1.1$ & $615.5 \mathrm{p}$ \\
\hline 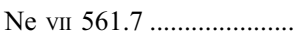 & $65.6 \pm 6.3$ & $47.3 \pm 0.7$ & $18.1 \pm 0.4$ & $104.7 \mathrm{~s}$ \\
\hline 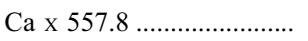 & $55.3 \pm 2.1$ & $65.5 \pm 1.4$ & $70.1 \pm 0.2$ & $251.6 \mathrm{r}$ \\
\hline 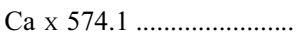 & $26.1 \pm 2.4$ & $37.7 \pm 0.1$ & $41.3 \pm 0.0$ & $152.6 \mathrm{r}$ \\
\hline 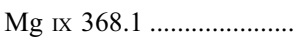 & $83.1 \pm 4.8$ & $186.4 \pm 2.8$ & $262.1 \pm 5.0$ & $1080.0 \mathrm{r}$ \\
\hline 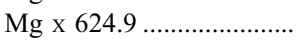 & $68.3 \pm 6.9$ & $233.1 \pm 1.7$ & $338.2 \pm 4.8$ & $1299.8 \mathrm{r}$ \\
\hline $\mathrm{Al}$ XI $550.0 \ldots \ldots \ldots \ldots . .$. & $37.2 \pm 0.3$ & $80.9 \pm 0.1$ & $107.3 \pm 1.6$ & $377.9 \mathrm{r}$ \\
\hline 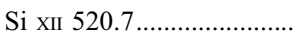 & $26.8 \pm 2.0$ & $175.5 \pm 1.7$ & $399.8 \pm 8.4$ & $1749.2 \mathrm{r}$ \\
\hline 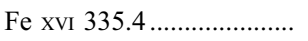 & $218.3 \pm 8.2$ & $869.2 \pm 15.1$ & $1876.7 \pm 30.8$ & $6775.8 \mathrm{r}$ \\
\hline
\end{tabular}

Notes.-All quantities in this table have the same meaning as in Table 1. Here, however, we have only two CDS rasters from which to calculate averages and their associated uncertainties. The $\mathrm{O}$ III line at $525.8 \AA$ and the Ne v line at $572.2 \AA$ both suffer from data flaws during the second raster scan on May 13. That is why we display the weaker Ne v line at $569.8 \AA$ in Figs. 1 and 2 . The $572.2 \AA$ image was cleaned enough that we include its average values and their associated uncertainties in this table. For O III at $525.8 \AA$ we include only data from the first raster scan for the average sunspot and average region intensities. 
We used the VLA to obtain radio brightness temperature maps in the right-hand $(R)$ and left-hand $(L)$ circularly polarized components at observing frequencies of $4.535 \mathrm{GHz}$ $\left(R_{5}, L_{5}\right), 8.065 \mathrm{GHz}\left(R_{8}, L_{8}\right)$, and $14.665 \mathrm{GHz}\left(R_{15}, L_{15}\right)$. The VLA was in its most compact ("D") configuration, best suited for viewing large, extended structures. Its spatial resolution (beam width) was about $15^{\prime \prime}, 10^{\prime \prime}$, and $5^{\prime \prime}$, respectively, at the above frequencies. In order to account for the quiet-Sun radio brightness temperature background (Zirin, Baumert, \& Hurford 1991), we added a constant value of $1.67 \times 10^{4} \mathrm{~K}$ to $R_{5}$ and $L_{5}, 1.26 \times 10^{4} \mathrm{~K}$ to $R_{8}$ and $L_{8}$, and $1.14 \times 10^{4} \mathrm{~K}$ to $R_{15}$ and $L_{15}$. The intensity (Stokes $I$ ) at any given radio frequency $\nu$ is given by $I_{\nu}=\left(R_{\nu}+L_{\nu}\right) / 2$, while the polarization (Stokes $V$ ) is given by $V_{\nu}=\left(R_{\nu}-L_{\nu}\right) / 2$. The absolute pointing of the VLA is well determined, so that all of the radio maps in our analysis were readily co-aligned with the EIT, MDI, and CDS data. The uncertainty in the co-alignment of all of our observations is $\sim 5^{\prime \prime}$.

\section{RESULTS}

Based on the present observations, we suggest that a sunspot plume may be defined as an EUV-emitting source, located above the photospheric sunspot, within which the spatially averaged intensities of emission lines formed at $5.2 \leq \log T \leq 5.7$ are enhanced by factors $\gtrsim 2$ relative to their respective average intensities within a $2^{\prime} \times 2^{\prime}$ area immediately surrounding the sunspot. (See Tables 1 and 2.) Although this may not be the ideal, "final" definition of a sunspot plume, it is more general than that proposed by either Foukal et al. (1974) or Brynildsen et al. (1999). Note, in particular, that it allows for the possibility that plumes may be seen well separated from their photospheric sunspots when they are observed in projection at the limb. We expect that, as plumes are observed more extensively and become better understood, definitions will evolve to incorporate their physical nature rather than their observed properties.

Figures 1 and 2 show $2^{\prime} \times 2^{\prime}$ average EUV intensity images of 12 emission lines observed on May 9 (Fig. 1) and 13 (Fig. 2), including $\mathrm{He}$ I at 537.1 $\AA, \mathrm{O}$ III 599.6, O IV 554.5 (a blend of four O Iv lines), O v 629.7, Ne v 569.8, Ne vi 562.8, Ne vII 561.7, Ca x 557.8, Mg x 624.9, Al XI 550.0, Si XII 520.7, and Fe XVI at $335.4 \AA$. The temperatures at which these lines are formed are listed in Table 1 . The umbral and penumbral boundaries of the sunspot, displayed as dashed contours, were obtained from the co-aligned MDI continuum images. Note that the $\mathrm{O}$ Iv, $\mathrm{O} v, \mathrm{Ne} v, \mathrm{Ne}$ vi, and $\mathrm{Ne}$ vir lines (and, to a lesser extent, the $\mathrm{Ca} \times$ line), formed at temperatures between $1.6 \times$ $10^{5}$ and $5.0 \times 10^{5} \mathrm{~K}$ (up to $7.9 \times 10^{5} \mathrm{~K}$ for $\mathrm{Ca} \mathrm{x}$ ), are relatively bright in the sunspot umbra, indicating an enhancement of transition region material at this location. This is consistent with the sunspot plume observations of Foukal et al. (1974), Fludra et al. (1997), Maltby et al. (1998), and Brynildsen et al. (1999). Since the O v line at $629.7 \AA$ is the most intense line at which the plume can be identified, we use it to outline the location of the plume in these and subsequent figures. Solid contours in each panel of Figures 1 and 2 display areas within which the intensity of the $\mathrm{O} v$ line exceeds $2500 \mathrm{ergs} \mathrm{cm}^{-2}$ $\mathrm{s}^{-1} \mathrm{sr}^{-1}$. (This value was chosen because it is intermediate between the average plume and average sunspot intensities of $\mathrm{O} v$ in Tables 1 and 2, and because it nicely outlines the area of enhanced $\mathrm{O} v$ plume emission in the figures.) The plume is clearly visible in the sunspot umbra on both dates, as are features of enhanced transition region emission just outside the sunspot penumbra. The latter appear brighter than the plume itself in $\mathrm{O}$ III and $\mathrm{O}$ IV, but fainter in $\mathrm{Ne} v, \mathrm{Ne}$ VI, and $\mathrm{Ne}$ VII.

Tables 1 and 2 list time-averaged intensities for the 12 lines displayed in Figures 1 and 2 (along with eight additional lines) for three different spatial features observed on May 9 and 13: the sunspot plume, the whole sunspot, and the entire $2^{\prime} \times 2^{\prime}$ area. Here the average intensities and their respective uncertainties were calculated to cover the ranges of (spatially averaged feature) intensities observed within the CDS rasters on each date. For example, the minimum plume-averaged $\mathrm{O} v$ intensity observed in any of the five CDS rasters on May 9 is the average intensity listed in Table 1 (3323.8) minus its associated uncertainty (434.2), or 2889.6 ergs $\mathrm{cm}^{-2} \mathrm{~s}^{-1} \mathrm{sr}^{-1}$. Similarly, the maximum plume-averaged $\mathrm{O} v$ intensity observed in any of the five CDS rasters on May 9 is 3758.0 ergs $\mathrm{cm}^{-2} \mathrm{~s}^{-1} \mathrm{sr}^{-1}$. This latter value is nearly equal to the plumeaveraged $\mathrm{O} v$ intensity observed on May 13 (3775.1), which has a very small associated uncertainty (3.8; possibly due to the fact that we have only two CDS rasters on the 13th). At any rate, the maximum plume-averaged intensity variation that we observe on and between May 9 and 13 is $\sim 13 \%$ of the May 9 average value, comparable to the plume intensity fluctuations reported by Noyes et al. (1985) as well as the amplitude of sunspot intensity oscillations reported by Fludra et al. (1997) and Fludra (2001). Based on O v intensities averaged over the whole sunspot, Tables 1 and 2 reveal maximum fluctuations (from the average value observed on May 9) $\sim 5 \%$. Thus, Figures 1 and 2 and Tables 1 and 2 indicate an overall stability of the plume (in both location and intensity) during our observing period.

Figures 3 and 4 show contour maps of the radio brightness temperatures at $4.535,8.065$, and $14.665 \mathrm{GHz}$ measured with the VLA on May 9 and 13. All six panels of each figure show the average $\mathrm{O} v$ intensity image along with the corresponding umbral and penumbral boundaries (dashed contours). Notice again that the plume is clearly visible within the sunspot umbra. The top three panels of each figure display radio brightness temperature contours of the total intensities $(I)$, and the bottom three panels show the polarizations $(V)$. Tables 3 and 4 list the average brightness temperatures of the $I, V, R$, and $L$ components in the plume, sunspot, and entire $2^{\prime} \times 2^{\prime}$ area for each observing frequency on each date. In addition, these tables list the maximum measured values of $I, V, R$, and $L$ within the $2^{\prime} \times 2^{\prime}$ area, along with their locations (in the plume, in the sunspot outside the plume, or somewhere in the region outside the spot). Third harmonic gyroemission at the above three radio observing frequencies corresponds to magnetic field strengths of 540,961, and $1748 \mathrm{G}$ in the radio source. Since the radio brightness temperatures indicate that the radio emission originates in the corona and transition region, these field strengths must be present in the corona and transition region. For example, since the maximum value for $R_{15}$ on May 13 is $1.6 \times 10^{6} \mathrm{~K}$, we know that field strengths of at least $1748 \mathrm{G}$ must be present in the corona on that date. Field strengths appropriate for second harmonic gyroemission at 4.535 and $8.065 \mathrm{GHz}(810$ and $1442 \mathrm{G})$, therefore, are also present in the corona. However, field strengths appropriate for second-harmonic gyroemission at $14.665 \mathrm{GHz}(2622 \mathrm{G})$, if present at all, must occur very low in the sunspot atmosphere, where the temperature is no more than several times $10^{4} \mathrm{~K}$.

MDI measured maximum photospheric longitudinal magnetic field strengths of +1570 and $+1700 \mathrm{G}$ in the umbra on May 9 and 13, respectively. These measured field strengths should be viewed with some caution, however, since a 

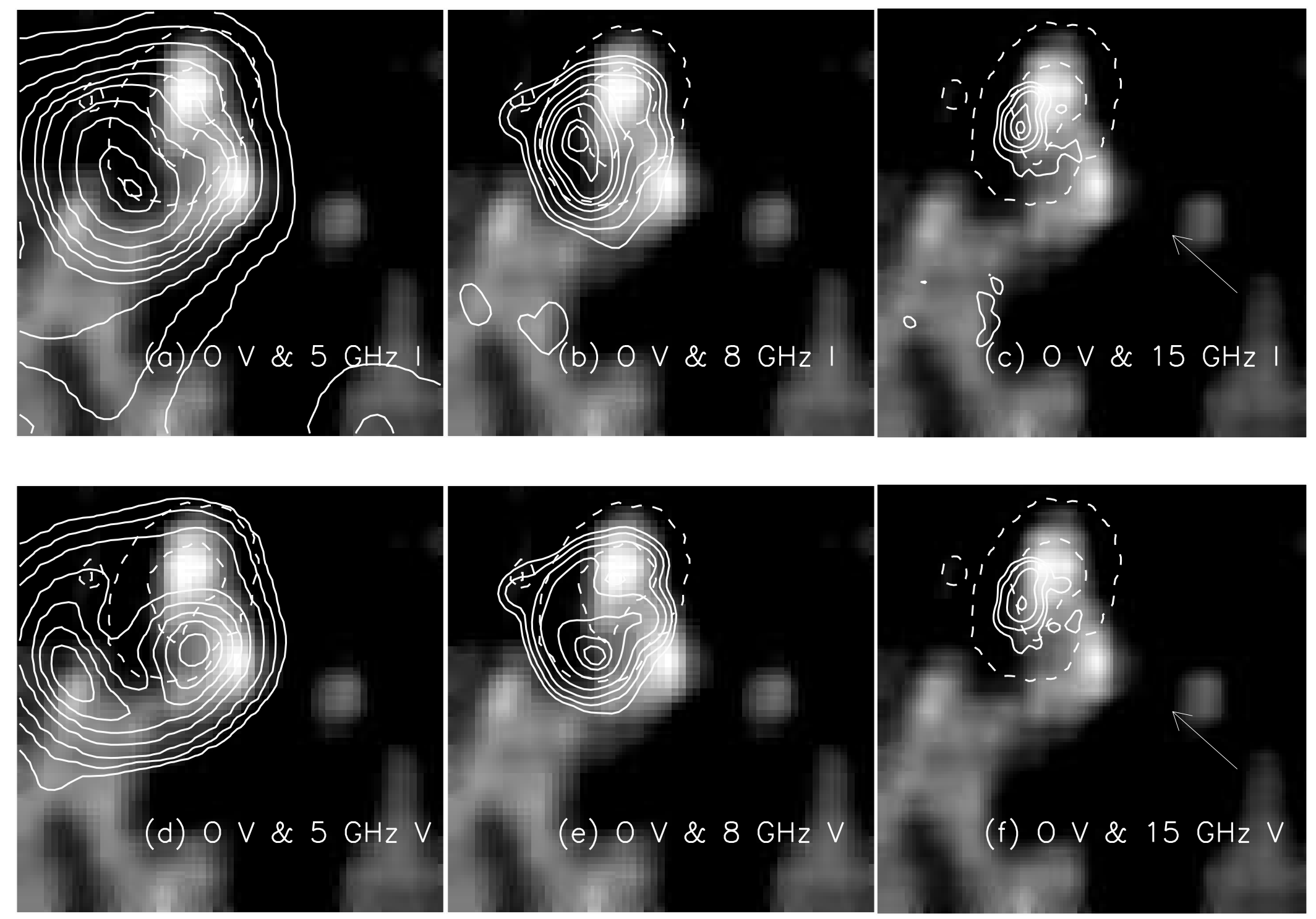

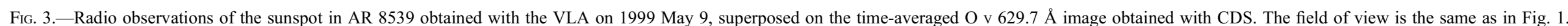

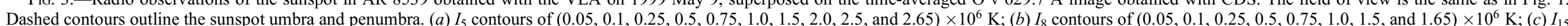

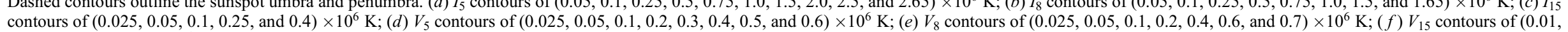

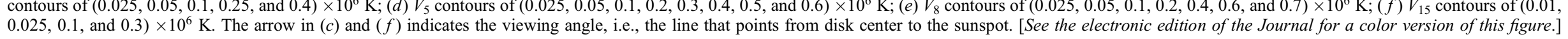



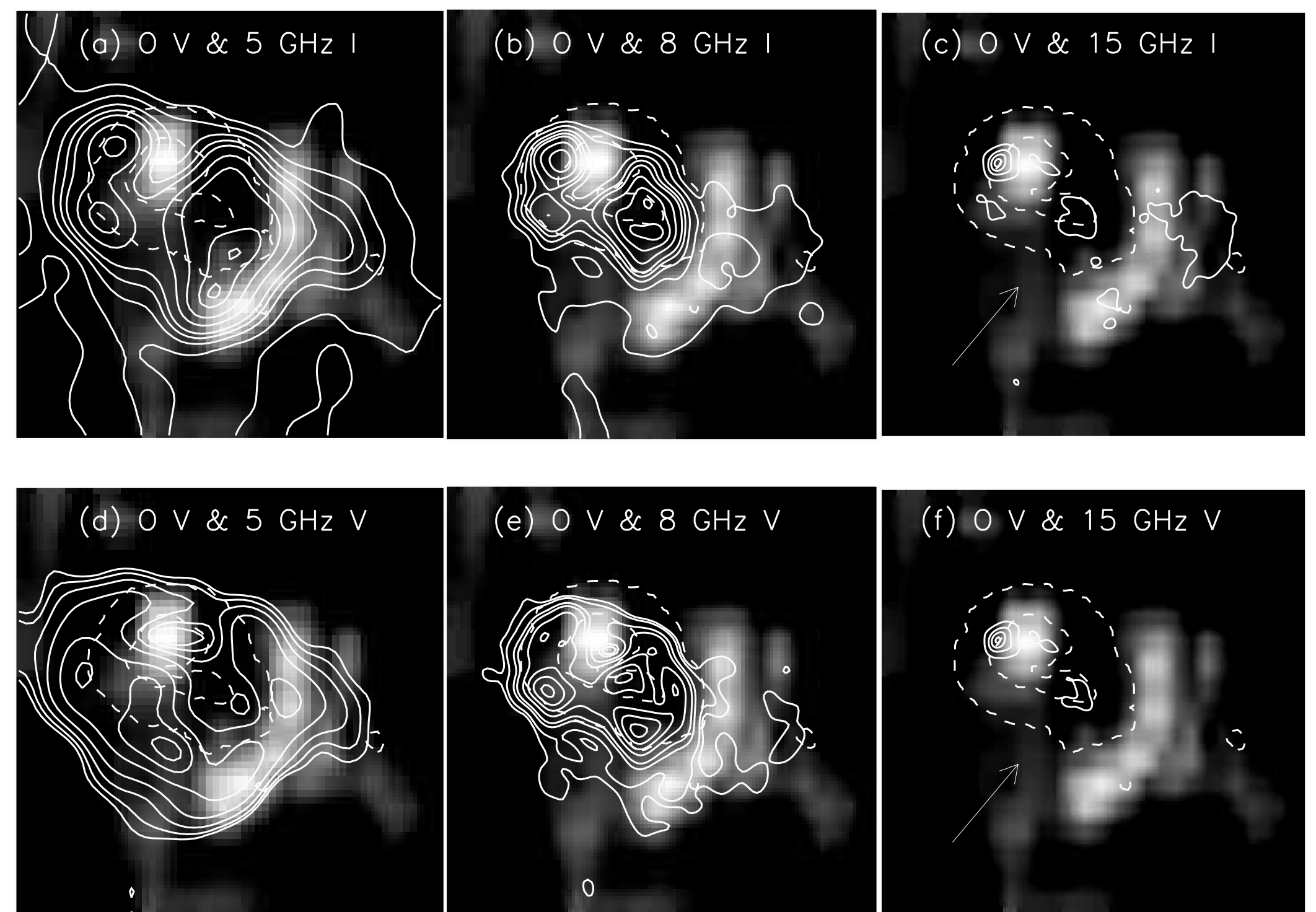

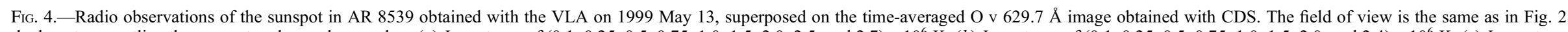

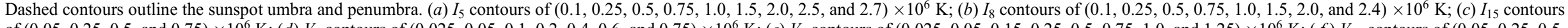

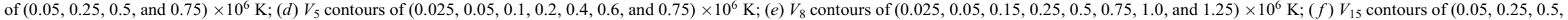

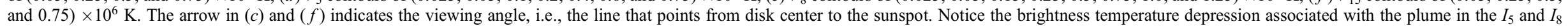
maps and the polarization depression associated with the plume in the $V_{5}$ and $V_{8}$ maps. [See the electronic edition of the Journal for a color version of this figure.] 
TABLE 3

Radio Brightness Temperatures in the Plume, Sunspot, and Surroundings

\begin{tabular}{|c|c|c|c|c|}
\hline \multirow{2}{*}{ QUANTITY } & \multicolumn{3}{|c|}{1999 May 9 Average Intensities } & \multirow[b]{2}{*}{ MAX } \\
\hline & Plume & Sunspot & Region & \\
\hline 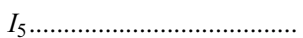 & $0.798 \times 10^{6}$ & $1.001 \times 10^{6}$ & $0.377 \times 10^{6}$ & $2.683 \times 10^{6} \mathrm{~s}$ \\
\hline 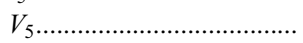 & $0.152 \times 10^{6}$ & $0.212 \times 10^{6}$ & $0.077 \times 10^{6}$ & $0.663 \times 10^{6} \mathrm{~s}$ \\
\hline 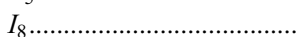 & $0.464 \times 10^{6}$ & $0.496 \times 10^{6}$ & $0.074 \times 10^{6}$ & $1.739 \times 10^{6} \mathrm{~s}$ \\
\hline$V_{8}$ & $0.145 \times 10^{6}$ & $0.204 \times 10^{6}$ & $0.029 \times 10^{6}$ & $0.770 \times 10^{6} \mathrm{~s}$ \\
\hline 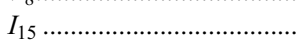 & $0.037 \times 10^{6}$ & $0.033 \times 10^{6}$ & $0.015 \times 10^{6}$ & $0.452 \times 10^{6} \mathrm{~s}$ \\
\hline 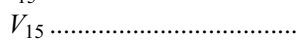 & $0.025 \times 10^{6}$ & $0.017 \times 10^{6}$ & $0.002 \times 10^{6}$ & $0.342 \times 10^{6} \mathrm{~s}$ \\
\hline 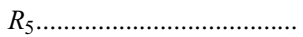 & $0.949 \times 10^{6}$ & $1.213 \times 10^{6}$ & $0.454 \times 10^{6}$ & $2.958 \times 10^{6} \mathrm{~s}$ \\
\hline$L_{5}$ & $0.646 \times 10^{6}$ & $0.789 \times 10^{6}$ & $0.299 \times 10^{6}$ & $2.408 \times 10^{6} \mathrm{~s}$ \\
\hline 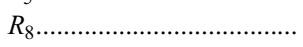 & $0.622 \times 10^{6}$ & $0.713 \times 10^{6}$ & $0.116 \times 10^{6}$ & $2.307 \times 10^{6} \mathrm{~s}$ \\
\hline 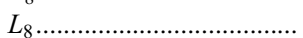 & $0.332 \times 10^{6}$ & $0.305 \times 10^{6}$ & $0.057 \times 10^{6}$ & $1.410 \times 10^{6} \mathrm{~s}$ \\
\hline$R_{15}$ & $0.062 \times 10^{6}$ & $0.050 \times 10^{6}$ & $0.017 \times 10^{6}$ & $0.794 \times 10^{6} \mathrm{~s}$ \\
\hline 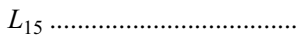 & $0.012 \times 10^{6}$ & $0.016 \times 10^{6}$ & $0.013 \times 10^{6}$ & $0.136 \times 10^{6} \mathrm{~s}$ \\
\hline
\end{tabular}

Notes.-Radio brightness temperatures are in K. Average sunspot intensities include the plume, and average region intensities include the sunspot. The maximum intensity ("Max") corresponds to the maximum value measured in any VLA spatial pixel within the 2 ' $\times 2$ ' FOV; "p" means "in the plume," "s" means "in the sunspot," and "r" means "in the region."

cross-calibration between MDI and the Advanced Stokes Polarimeter (ASP) reveals that MDI may underestimate sunspot field strengths by factors $\sim 0.69$ (Berger \& Lites 2003; see also Green et al. 2003). ASP data exist for AR 8539 on May 13 (Leka et al. 2001) and are consistent with such a factor: they show a peak longitudinal surface field strength of $+2300 \mathrm{G}$ and a peak total surface field strength of $2800 \mathrm{G}$.

Although dipole sunspot models and various extrapolations indicate that coronal magnetic field strengths are expected to be greatest over the center of the umbra, none of the radio brightness temperature peaks occur there on either of our observing dates. For a dipole sunspot viewed at some location away from disk center, projection effects and angular effects are expected to produce a shift in the location of the peak brightness temperature toward the limb, with a brightness temperature depression expected on the side of the spot closest to disk center (Gelfreikh \& Lubyshev 1979; Brosius \& Holman 1989). However, this is not what we see on either of our observing dates. On May 9, when the sunspot was located in the northeast (upper left) quadrant of the solar disk (at $x=-450^{\prime \prime}, y=+400^{\prime \prime}$ ), the brightness temperature peaks were expected to be displaced toward the north and east in Figure 3 (up and left, along the direction of the arrow in Figs. $3 c$ and $3 f$ ). The observed displacement in peak location is along a line perpendicular to this, toward the south and east. No depression in the brightness temperature is observed on this date. (It is worth mentioning that the shifts in the radio brightness temperature peaks from the center of the umbra are much greater than the $\sim 5^{\prime \prime}$ uncertainty in the co-alignment.)

Similar effects are seen on May 13, when the sunspot was located in the northwest (upper right) quadrant of the solar disk (at $x=+320^{\prime \prime}, y=+380^{\prime \prime}$ ). The ASP data (Leka et al. 2001 ) indicate that the magnetic field in the large sunspot was close to potential on this date. The structure of the radio emission is more complex on this date than it is on May 9, but again, none of the brightness temperature centroids exhibit the theoretically expected displacement toward the north and west in Figure 4 (up and right, along the direction of the arrow in

TABLE 4

Radio Brightness Temperatures in the Plume, Sunspot, and Surroundings

\begin{tabular}{|c|c|c|c|c|}
\hline \multirow[b]{2}{*}{ QUANTITY } & \multicolumn{3}{|c|}{1999 May 13 Average Intensities } & \multirow[b]{2}{*}{ MAX } \\
\hline & Plume & Sunspot & Region & \\
\hline ..................... & $0.668 \times 10^{6}$ & $1.493 \times 10^{6}$ & $0.463 \times 10^{6}$ & $2.808 \times 10^{6} \mathrm{r}$ \\
\hline ........................ & $0.061 \times 10^{6}$ & $0.237 \times 10^{6}$ & $0.092 \times 10^{6}$ & $0.838 \times 10^{6} \mathrm{r}$ \\
\hline 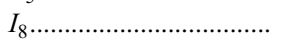 & $0.528 \times 10^{6}$ & $0.888 \times 10^{6}$ & $0.154 \times 10^{6}$ & $2.535 \times 10^{6} \mathrm{~s}$ \\
\hline 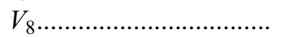 & $0.184 \times 10^{6}$ & $0.437 \times 10^{6}$ & $0.064 \times 10^{6}$ & $1.338 \times 10^{6} \mathrm{~s}$ \\
\hline 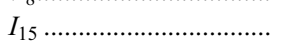 & $0.064 \times 10^{6}$ & $0.034 \times 10^{6}$ & $0.017 \times 10^{6}$ & $0.841 \times 10^{6} \mathrm{~s}$ \\
\hline 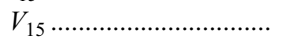 & $0.068 \times 10^{6}$ & $0.024 \times 10^{6}$ & $0.001 \times 10^{6}$ & $0.797 \times 10^{6} \mathrm{~s}$ \\
\hline 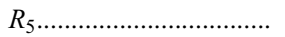 & $0.729 \times 10^{6}$ & $1.730 \times 10^{6}$ & $0.555 \times 10^{6}$ & $3.243 \times 10^{6} \mathrm{r}$ \\
\hline$L_{5} \ldots \ldots \ldots \ldots \ldots \ldots \ldots \ldots \ldots \ldots \ldots \ldots \ldots \ldots \ldots \ldots \ldots \ldots$ & $0.607 \times 10^{6}$ & $1.256 \times 10^{6}$ & $0.370 \times 10^{6}$ & $2.573 \times 10^{6} \mathrm{~s}$ \\
\hline 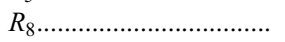 & $0.711 \times 10^{6}$ & $1.325 \times 10^{6}$ & $0.217 \times 10^{6}$ & $3.335 \times 10^{6} \mathrm{~s}$ \\
\hline 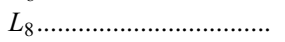 & $0.344 \times 10^{6}$ & $0.452 \times 10^{6}$ & $0.090 \times 10^{6}$ & $1.957 \times 10^{6} \mathrm{~s}$ \\
\hline 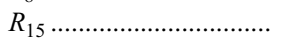 & $0.131 \times 10^{6}$ & $0.057 \times 10^{6}$ & $0.019 \times 10^{6}$ & $1.638 \times 10^{6} \mathrm{~s}$ \\
\hline$L_{15} \ldots \ldots \ldots \ldots \ldots \ldots \ldots \ldots \ldots \ldots$ & $\ldots$ & $0.010 \times 10^{6}$ & $0.016 \times 10^{6}$ & $0.066 \times 10^{6} \mathrm{r}$ \\
\hline
\end{tabular}

Notes.-Radio brightness temperatures are in K. Average sunspot intensities include the plume, and average region intensities include the sunspot. The maximum intensity ("Max") corresponds to the maximum value measured in any VLA spatial pixel within the $2^{\prime} \times 2^{\prime}$ FOV; "p" means "in the plume," "s" means "in the sunspot," and " $r$ " means "in the region." 

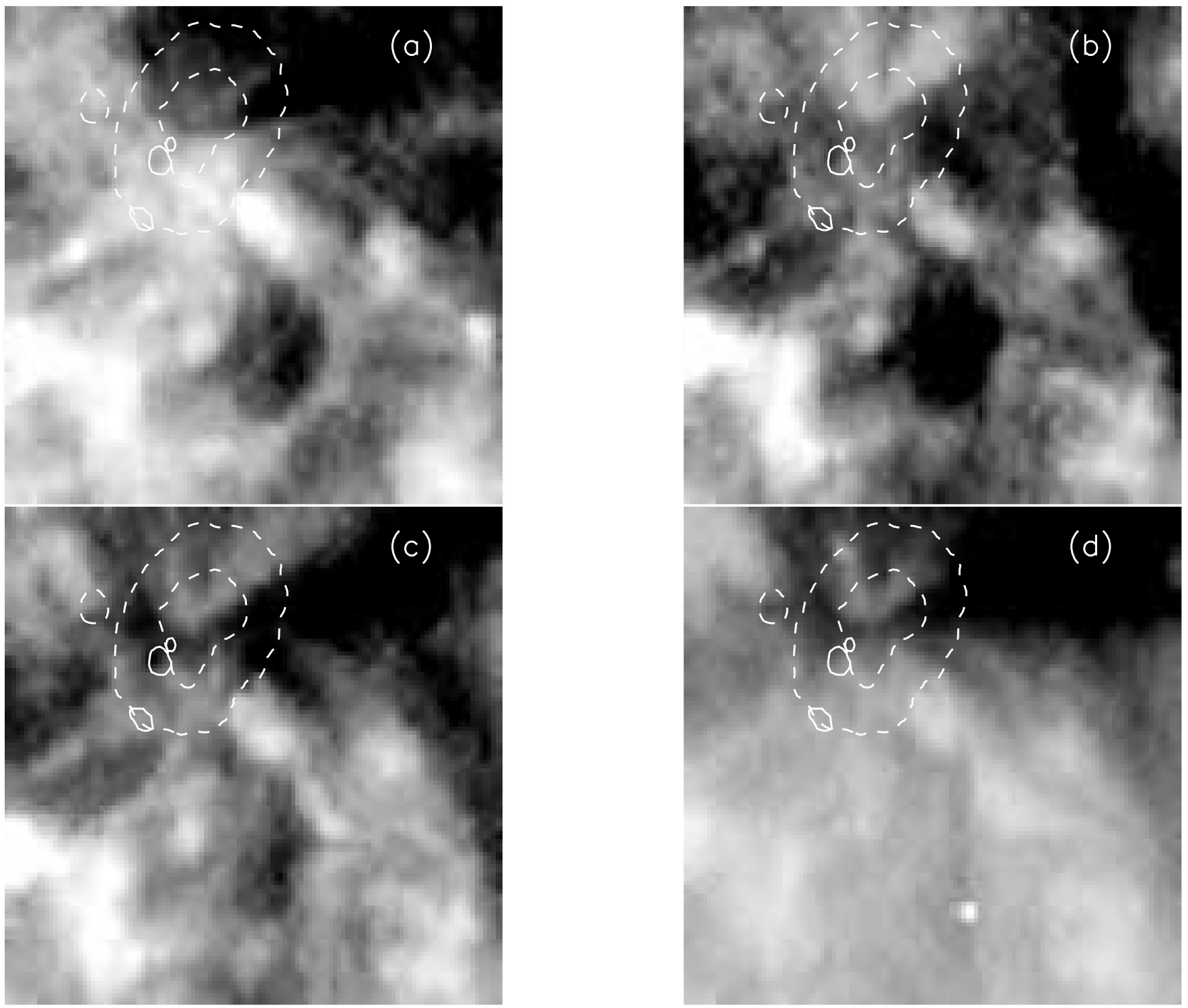

FIG. 5.- Images of the sunspot in AR 8539 in EUV emission lines of $(a)$ He II at $304 \AA$, (b) Fe IX/x at $171 \AA,(c)$ Fe XII at $195 \AA$, and $(d)$ Fe XV at $284 \AA$ obtained with EIT around 1900 UT on 1999 May 9. The field of view is the same as in Figs. 1 and 3. The sunspot umbra and penumbra boundaries are displayed as dashed contours. Solid contours correspond to $I_{15}=0.4 \times 10^{6} \mathrm{~K}$ (closest to spot center), $I_{8}=1.65 \times 10^{6} \mathrm{~K}$ (next farthest from spot center), and $I_{5}=2.65 \times 10^{6} \mathrm{~K}$ (farthest from spot center). [See the electronic edition of the Journal for a color version of this figure.]

Figs. $4 c$ and $4 f$ ). Rather, of the three peaks observed in the 4.535 and $8.065 \mathrm{GHz}$ intensity maps, one of them is displaced toward disk center while the other two are shifted along a line perpendicular to the expected shift. Here, however, we do observe a depression in the radio brightness temperature. It is not shifted, as would be theoretically expected, toward disk center, but occurs directly within the sunspot plume. Not only that, the average 4.535 and $8.065 \mathrm{GHz}$ brightness temperatures within the plume $\left(R_{5}, L_{5}, R_{8}\right.$, and $L_{8}$ in Table 4$)$ vary from $3.44 \times 10^{5}$ to $7.29 \times 10^{5} \mathrm{~K}\left(5.54 \leq \log T_{B} \leq 5.86\right)$, comparable to the formation temperatures of prominent plume lines. We conclude that the radio brightness temperature depression observed in the 4.535 and $8.065 \mathrm{GHz}$ emission on 1999 May 13 is due to thermal gyroemission from the sunspot plume.

Although no depression is evident in the 4.535 and $8.065 \mathrm{GHz}$ maps of May 9, the average brightness temperatures within the plume $\left(R_{5}, L_{5}, R_{8}\right.$, and $L_{8}$ in Table 3$)$ vary from $3.32 \times 10^{5}$ to $9.49 \times 10^{5} \mathrm{~K}\left(5.52 \leq \log T_{B} \leq 5.98\right)$, similar to the values above for May 13. Thus, the radio emission from the sunspot umbra on both May 9 and 13 is dominated by thermal gyroemission from the plume.

An additional feature of our observations is the small, intense, highly polarized $14.665 \mathrm{GHz}$ source located near the eastern edge of the umbra on both dates. The fact that this source lies not over the center of the umbra (where the largest photospheric field strengths occur), but is displaced far from it, indicates that the magnetic field over the spot is not symmetric. A similar $15 \mathrm{GHz}$ source was reported by White, Kundu, \& Gopalswamy (1991) from one day of high-dynamic range VLA observations. Here, we find that the $14.665 \mathrm{GHz}$ source persists for at least two observing days, indicating a stability in the asymmetry of the sunspot's coronal magnetic field.

Figure 5 shows $2^{\prime} \times 2^{\prime}$ images of $\mathrm{He}$ II at $304 \AA$, Fe IX/X at $171 \AA, \mathrm{Fe}$ XII at $195 \AA$, and Fe XV at $284 \AA$ obtained with EIT on May 9. These lines are formed at temperatures around 

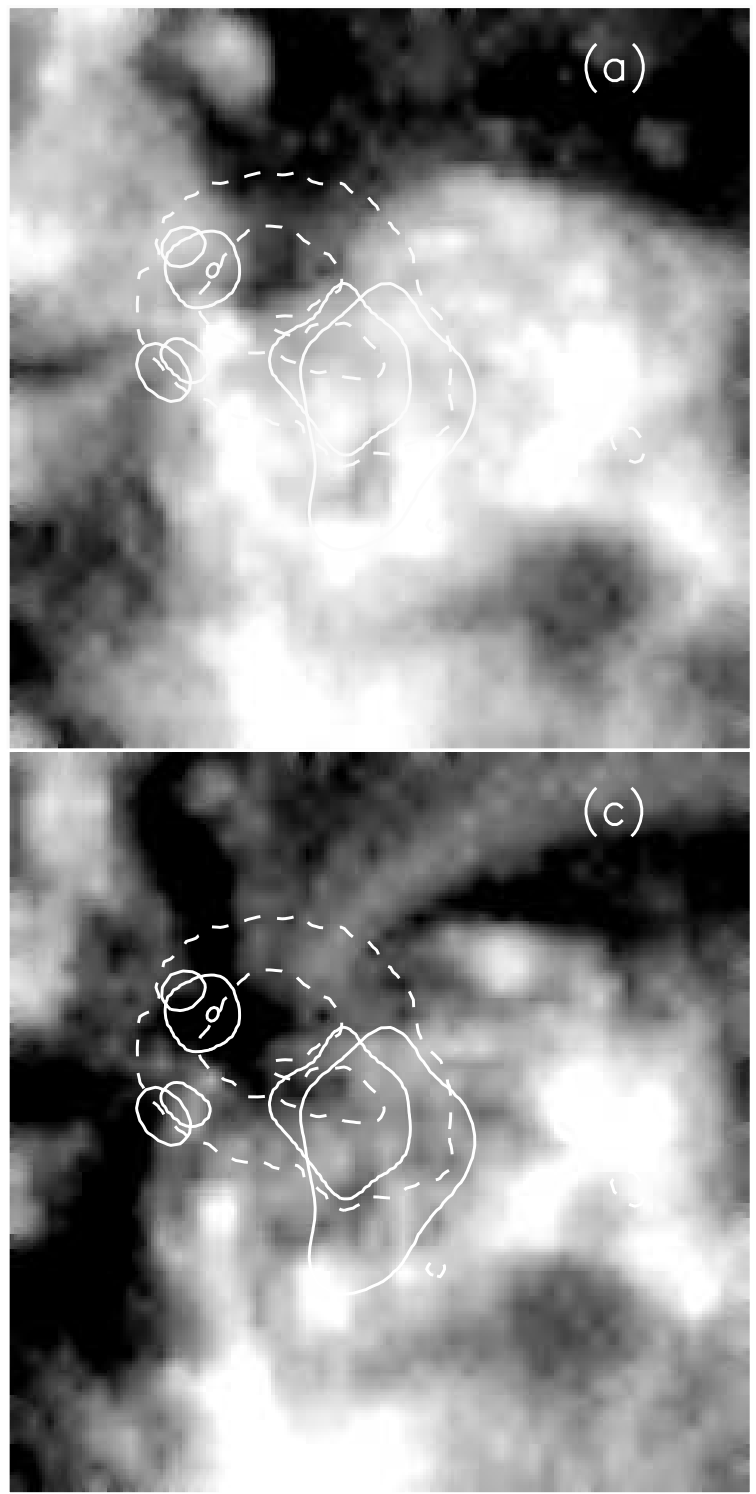
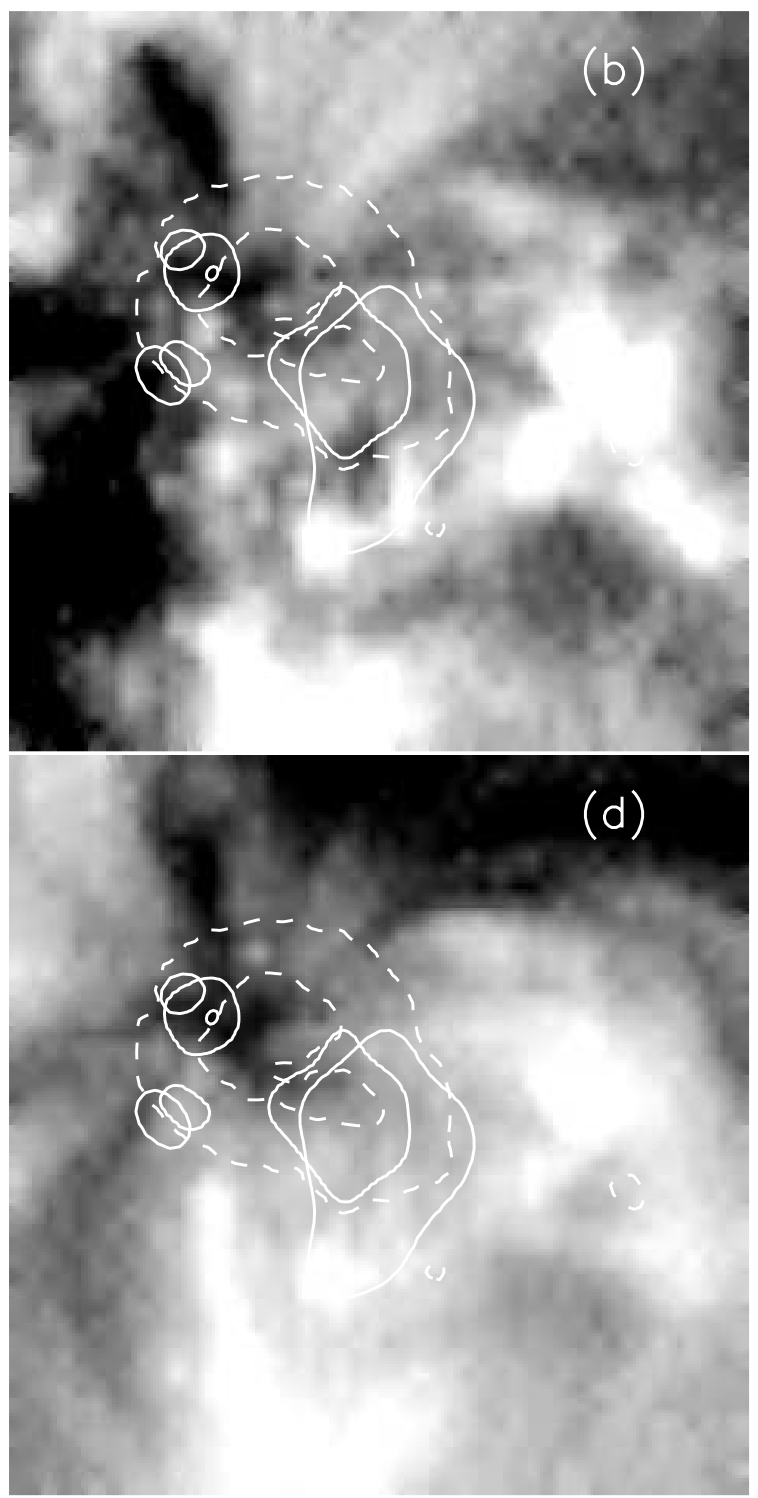

FIG. 6. - Images of the sunspot in AR 8539 in EUV emission lines of (a) He II at $304 \AA$, (b) Fe IX/X at $171 \AA,(c)$ Fe XII at $195 \AA$, and $(d)$ Fe xV at $284 \AA$ obtained with EIT around 1900 UT on 1999 May 13. The field of view is the same as in Figs. 2 and 4. The sunspot umbra and penumbra boundaries are displayed as dashed contours. Solid contours correspond to $I_{15}=0.75 \times 10^{6} \mathrm{~K}$ (closest to spot center), $I_{8}=1.2 \times 10^{6} \mathrm{~K}$ (next farthest from spot center), and $I_{5}=2.0 \times 10^{6} \mathrm{~K}$ (farthest from spot center). Contours of all three frequencies are found only in the northeast portion of the spot; the other two contour pairs include only $I_{8}$ and $I_{5}$. [See the electronic edition of the Journal for a color version of this figure.]

$4.7 \times 10^{4}, 1.0 \times 10^{6}, 1.4 \times 10^{6}$, and $2.0 \times 10^{6} \mathrm{~K}$, respectively. The field of view (FOV) for each panel is the same as in Figures 1 and 3, and the umbral and penumbral boundaries are again plotted as dashed contours. Intensities in these images are displayed on a logarithmic scale, and reveal the presence of faint coronal emission within both the umbra and the penumbra, consistent with the CDS measurement of weak coronal line emission there (Table 1). One contour level for each of $I_{5}, I_{8}$, and $I_{15}$ is plotted in all four panels, showing the locations of the radio brightness temperature peaks. The $I_{15}$ source appears closest to spot center near the umbral boundary, followed by the $I_{8}$ and $I_{5}$ sources. Notice that the $I_{15}$ source appears in one of the darkest EUV portions of the sunspot, indicating that the magnetic flux tube containing the largest coronal magnetic field strengths has a comparatively low emission measure. Figure 6 is similar to Figure 5, but for May 13. Here, in addition to the closely-spaced sequence of $I_{5}, I_{8}$, and $I_{15}$ sources in the upper left of the spot (between the umbral and penumbral boundaries), we see two additional pairs of $I_{5}$ and $I_{8}$ contours. In each of those two cases, the $I_{8}$ source lies closer to spot center than does the $I_{5}$ source. As with the May 9 observations, the $I_{15}$ source appears in one of the darkest EUV portions of the sunspot.

\section{DISCUSSION}

Our sunspot umbra appears relatively dark in EUV line emission formed at coronal temperatures (Figs. 1, 2, 5, and 6). It does, however, emit a small but measurable amount of radiation in those lines (Tables 1 and 2). This is consistent with the observations of Strong et al. (1984), who observed weak soft X-ray emission in the umbra above a sunspot with the $S M M$ XRP. They concluded, based on the brightness temperature depression observed in their $5 \mathrm{GHz}$ radio observations, that the radio emission from the sunspot umbra originated in a volume of cool plasma, possibly associated with a sunspot plume. While Strong et al. (1984) could only infer the 
presence of that cooler plasma in their sunspot, we directly, unambiguously observe it in strong EUV line emission from our sunspot. Thus, both our observations and theirs indicate that magnetic field strengths appropriate for gyroemission occur inside the sunspot plume, not in the hot, low intensity, coronal plasma outside (probably above) the plume.

In contrast to this, Lang \& Willson (1982) reported that the peak in their $5 \mathrm{GHz}$ radio observations of a sunspot occurred over the umbra, and that their observed radio brightness temperatures were generally well correlated with the measured photospheric longitudinal magnetic field strength. Zlotnik et al. (1996) also observed ring structure from a sunspot near disk center that can be well fitted by simple symmetric coronal models. While these results are certainly different from what we find here, it simply means that the radio source that they observed in the sunspot umbra was embedded in plasma at coronal temperatures, not in (plume) plasma at transition region temperatures.

One goal of multifrequency radio gyroemission observations is to determine the magnetic field and temperature structure of coronal magnetic loops (e.g., Holman \& Kundu 1985). For example, Brosius et al. (1992) used a series of radio brightness temperature centroids to outline the loop structure connecting a sunspot to its trailing plage. In the present work, however, it is not obvious that the radio brightness temperature centroids shown in Figures 5 and 6 outline any particular EUV loop or structure in the region. Recall, for example, that the $I_{15}$ sources lie in the darkest EUV portions of the sunspot, where they do not overlie any EUV counterparts. Similarly, the $I_{8}$ and $I_{5}$ sources nearest the $I_{15}$ source on May 13 occur within a region of EUV depletion. It is possible, however, that the two southern pairs of $I_{8}$ and $I_{5}$ sources on May 13 correspond to coronal structures seen in the EUV. Further, it may be that magnetic flux tubes such as the one revealed by the $I_{15}$ source correspond to compact, soft X-ray flux tubes like those reported by Sams, Golub, \& Weiss (1992) based on Normal Incidence X-Ray Telescope (NIXT) observations obtained during a sounding rocket flight. We examined Yohkoh Soft X-Ray Telescope (SXT) images from May 9 and 13, but found no soft X-ray counterpart to the $I_{15}$ sources on either date. This is consistent with the sunspot survey study of Nindos et al. (2000), which is based on coordinated Yohkoh SXT and $17 \mathrm{GHz}$ radio observations from the Nobeyama Radioheliograph.

Coordinated EUV and radio observations such as the ones presented here help us gain a better understanding of the strength and structure of coronal magnetic fields in sunspots and active regions. Further analysis of this interesting data set will include a detailed derivation of the three dimensional coronal magnetic field (Brosius et al. 1997, 2002; Brosius 2004; Gary \& Lee 2003) and comparisons with extrapolations from photospheric vector magnetograms obtained with the ASP. It is expected that three-dimensional coronal magnetography will become routine with the advent of the Frequency Agile Solar Radiotelescope (FASR).

\section{SUMMARY}

We obtained coordinated VLA, CDS, EIT, and MDI observations of a large sunspot in NOAA Region 8539 on 1999 May 9 and 13. Based on the EUV observations, a sunspot plume is clearly visible in the umbra on both observing dates, and exhibits an overall stability in both location and intensity during our observing period. The plume appears brightest in emission lines formed at temperatures between $1.6 \times 10^{5}$ and $5.0 \times 10^{5} \mathrm{~K}$ (up to $7.9 \times 10^{5} \mathrm{~K}$ ). The radio emission from the sunspot umbra is dominated by thermal gyroemission from the plume, which accounts for radio brightness temperatures $<1 \times 10^{6} \mathrm{~K}$ in the umbra on both dates, as well as a brightness temperature depression in the umbra on May 13. A compact $14.665 \mathrm{GHz}$ source persists during our observing period, indicating a long-lived, compact flux tube connecting to the sunspot umbra/penumbra boundary. It occurs in a portion of the sunspot that appears very dark in EUV emission and indicates a coronal magnetic field strength of at least 1748 G. Research like this that depends on well-calibrated high spatial resolution radio observations will benefit from future observations with the Frequency Agile Solar Radiotelescope (FASR).

J. W. B. gratefully acknowledges NASA support through the Guest Investigator Program (grant NAG5-11757). Solar research at the University of Maryland is supported by NSF grant ATM 02-33907 and NASA grants NAG5-10175, NAG5-12732, and NAG5-12860. We thank the anonymous referee for valuable comments that helped improve the manuscript. The Very Large Array is run by the National Radio Astronomy Observatory, which is a facility of the National Science Foundation operated under cooperative agreement by Associated Universities, Inc.
Alissandrakis, C. E., \& Kundu, M. R. 1982, ApJ, 253, L49

Alissandrakis, C. E., Kundu, M. R., \& Lantos, P. 1980, A\&A, 82, 30

Berger, T. E., \& Lites, B. W. 2003, Sol. Phys., 213, 213

Brosius, J. W. 2004, in Solar and Space Weather Radiophysics, ed. D. E. Gary \& C. O. Keller (Dordrecht: Kluwer), in press

Brosius, J. W., Davila, J. M., Thomas, R. J., \& White, S. M. 1997, ApJ, 488, 488

Brosius, J. W., \& Holman, G. D. 1989, ApJ, 342, 1172

Brosius, J. W., Landi, E., Cook, J. W., Newmark, J. S., Gopalswamy, N., \& Lara, A. 2002, ApJ, 574, 453

Brosius, J. W., Willson, R. F., Holman, G. D., \& Schmelz, J. T. 1992, ApJ, 386, 347

Brosius, J. W., et al. 1993, ApJ, 411, 410

Brynildsen, N., Maltby, P., Brekke, P., Haugan, S. V. H., \& Kjeldseth-Moe, O. 1999, Sol. Phys., 186, 141

Dere, K. P., Landi, E., Mason, H. E., Monsignori-Fossi, B. C., \& Young, P. R. 1997, A\&AS, 125, 149

Fludra, A. 2001, A\&A, 368, 639

Fludra, A., Brekke, P., Harrison, R. A., Mason, H. E., Pike, C. D., Thompson, W. T., \& Young, P. R. 1997, Sol. Phys., 175, 487
REFERENCES

Foukal, P. V. 1976, ApJ, 210, 575

Foukal, P. V., Huber, M. C. E., Noyes, R. W., Reeves, E. M., Schmahl, E. J., Timothy, J. G., Vernazza, J. G., \& Withbroe, G. L. 1974, ApJ, 193, L143

Gary, D. E., \& Hurford, G. J. 1987, ApJ, 317, 522

Gary, D. E., \& Lee, J. 2003, BAAS, 35, 847

Gelfreikh, G. B., \& Lubyshev, B. I. 1979, Soviet Astron., 23, 316

Gopalswamy, N., Raulin, J. P., Kundu, M. R., Hildebrandt, J., Kruger, A., \& Hofmann, A. 1996, A\&A, 316, L25

Green, L. M., Demoulin, P., Mandrini, C. H., \& van Driel-Gesztelyi, L. 2003, Sol. Phys., 215, 307

Harrison, R. A., et al. 1995, Sol. Phys., 162, 233

Holman, G. D., \& Kundu, M. R. 1985, ApJ, 292, 291

Kundu, M. R. 1965, Solar Radio Astronomy (New York: Wiley)

Landi, E., Landini, M., Dere, K. P., Young, P. R., \& Mason, H. E. 1999, A\&AS, 135,339

Lang, K. R., \& Willson, R. F. 1982, ApJ, 255, L111

Lee, J., McClymont, A. N., Mikic, Z., White, S. M., \& Kundu, M. R. 1998, ApJ, 501, 853

Leka, K. D., White, S., Mikic, Z., \& Lee, J. 2001, Eos, 82, S313 
Maltby, P., Brynildsen, N., Brekke, P., Haugan, S. V. H., Kjeldseth-Moe, O., \& Wikstol, O. 1998, ApJ, 496, L117

Mazzotta, P., Mazzitelli, G., Colafrancesco, S., \& Vittorio, N. 1998, A\&AS, 133,403

Nindos, A., Alissandrakis, C. E., Gelfreikh, G. B., Kundu, M. R., Dere, K. P., Korzhavin, A. N., \& Bogod, V. M. 1996, Sol. Phys., 166, 55

Nindos, A., Kundu, M. R., White, S. M., Shibasaki, K., \& Gopalswamy, N. 2000, ApJS, 130, 485

Noyes, R. W., Raymond, J. C., Doyle, J. G., \& Kingston, A. E. 1985, ApJ, 297, 805

Sams, B. J., Golub, L., \& Weiss, N. O. 1992, ApJ, 399, 313

Strong, K. T., Alissandrakis, C. E., \& Kundu, M. R. 1984, ApJ, 277, 865

Vourlidas, A., Bastian, T. S., \& Aschwanden, M. J. 1997, ApJ, 489, 403

White, S. M. 1999, Sol. Phys., 190, 309

2004, in Solar and Space Weather Radiophysics, ed. D. E. Gary \& C.

O. Keller (Dordrecht: Kluwer), in press
White, S. M., \& Kundu, M. R. 1997, Sol. Phys., 174, 31

White, S. M., Kundu, M. R., \& Gopalswamy, N. 1991, ApJ, 366, L43 1992, ApJS, 78, 599

White, S. M., Thomas, R. J., Brosius, J. W., \& Kundu, M. R. 2000, ApJ, 534, L203

Young, P. R., Landi, E., \& Thomas, R. J. 1998, A\&A, 329, 291

Zhang, J., Kundu, M. R., White, S. M., Dere, K. P., \& Newmark, J. S. 2001, ApJ, 561, 396

Zheleznyakov, V. V. 1970, Radio Emission of the Sun and Planets, ed. J. S. Hey (Oxford: Pergamon)

Zirin, H., Baumert, B. M., \& Hurford, G. J. 1991, ApJ, 370, 779

Zlotnik, E. Ya., Kundu, M. R., \& White, S. M. 1996, Radiophys. Quantum Electron., 39, 255 